Supporting Information

\title{
Ethylenediamine-Enhanced Oxidation Resistivity of a Copper Surface during Water-Based Copper Nanowire Synthesis
}

\author{
Jahyun Koo, ${ }^{\dagger}$ Soonho Kwon, ${ }^{\dagger}$ Na Rae Kim, Kihyun Shin, and Hyuck Mo Lee* \\ Department of Materials Science and Engineering, KAIST, 291 Daehak-ro, Yuseong-gu, \\ Daejeon 34141, Republic of Korea \\ ${ }^{\dagger}$ These authors contributed equally.
}

Address correspondence to $\underline{\text { hmlee@kaist.ac.kr }}$ 


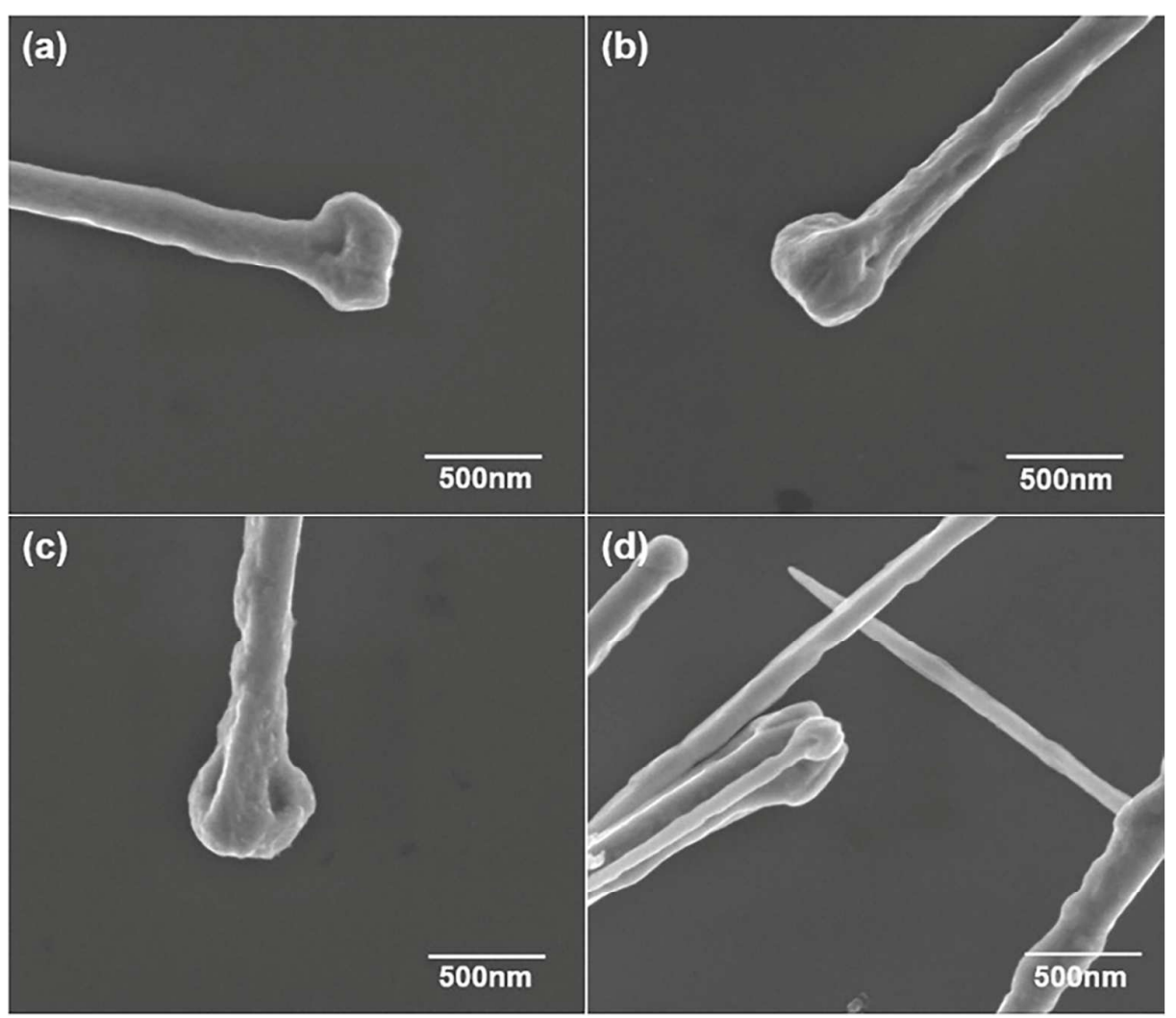

Figure S1. SEM image of $\mathrm{Cu}$ heads produced with $4 \mathrm{mmol}$ of EDA at the two-step reaction temperature. 

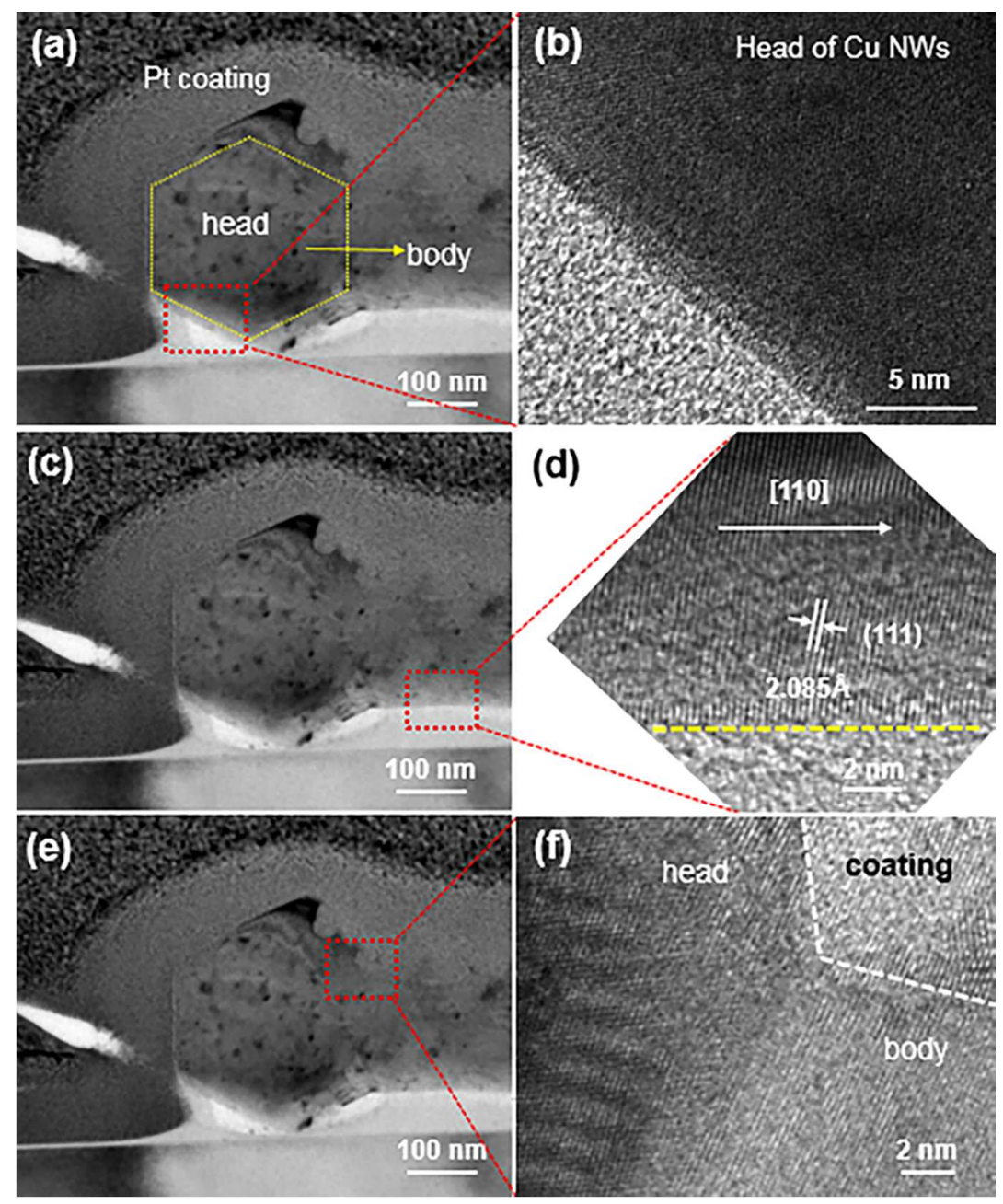

Figure S2. (a), (c), (e) TEM images of $\mathrm{Cu}$ NWs after FIB ion milling (perpendicular to the vertical axis) and (b), (d), (f) TEM images of the head, body and head-body intersection, respectively. 

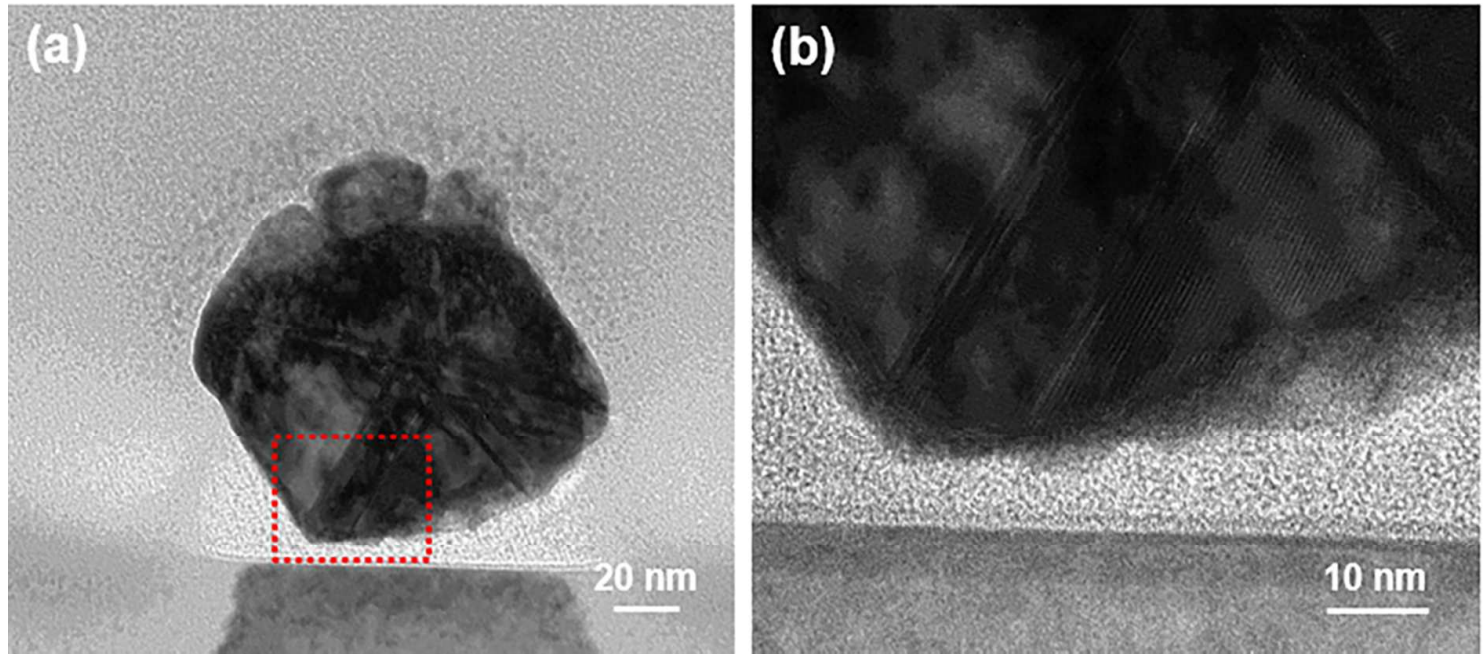

Figure S3. (a) TEM image of the body of a Cu NW after FIB ion milling (perpendicular to horizontal axis) and (b) a magnified TEM image. 


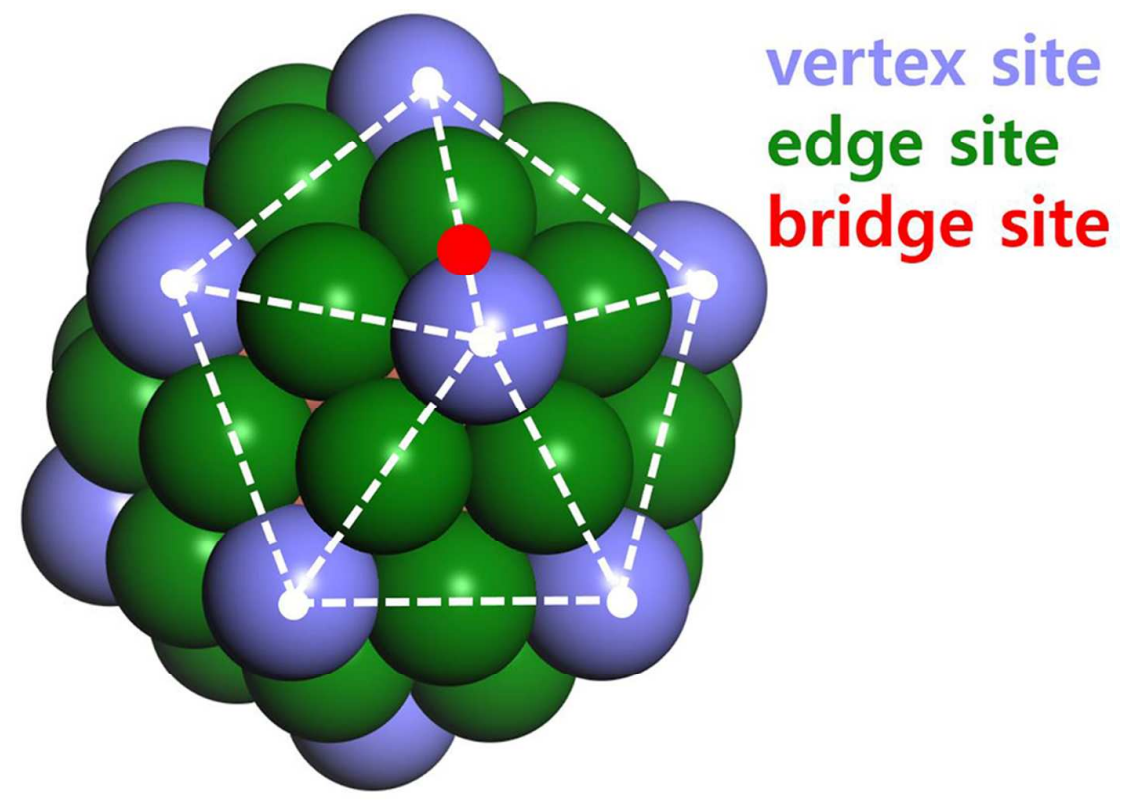

Figure S4. The adsorption sites on the $\mathrm{Cu}_{55}$ NP. The icosahedron, which consists of 55 atoms, only has vertex and edge surface atoms. 


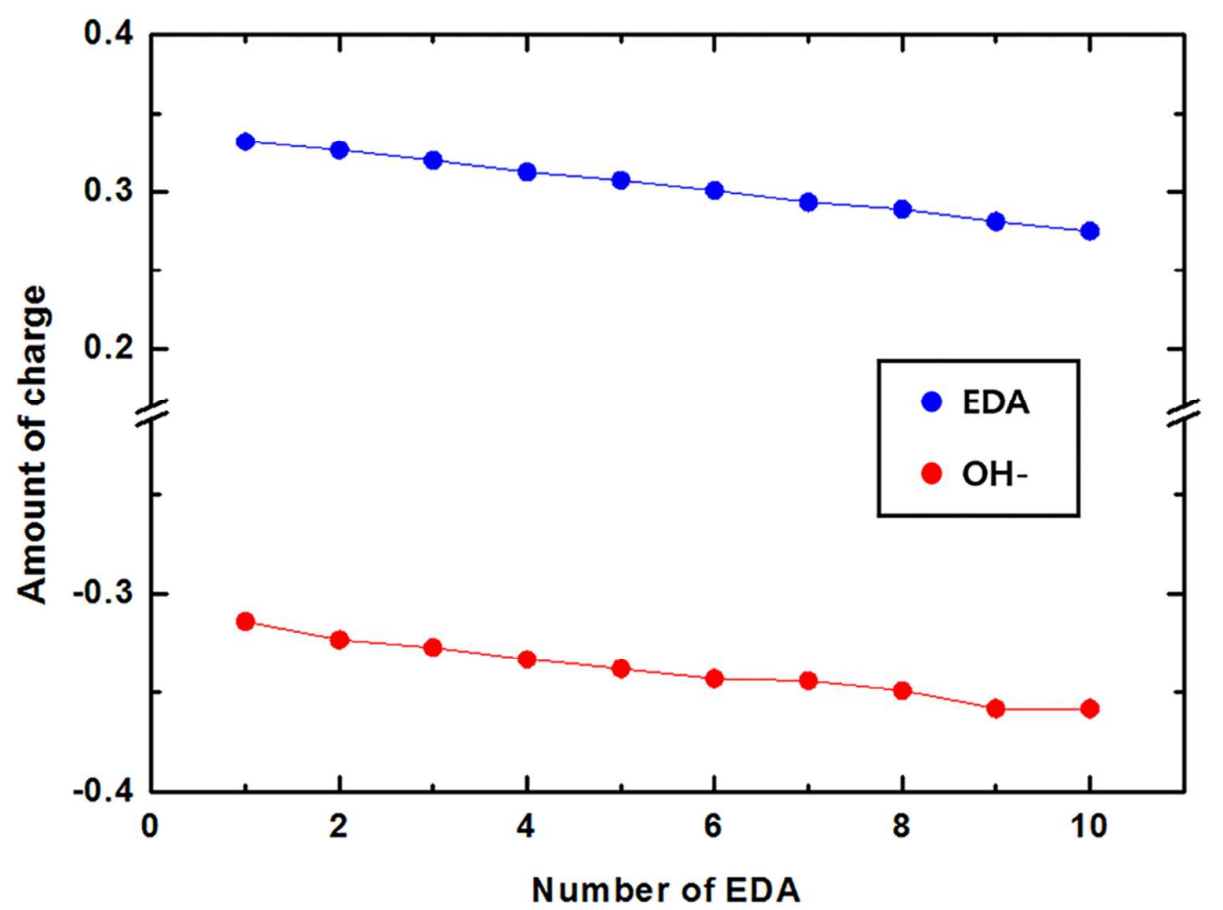

Figure S5. Mulliken charges of EDA and $\mathrm{OH}^{-}$molecules as a function of EDA coverage. 
Table S1. Relative energies of the decahedron (Dh) and cuboctahedron (Coh) compared to that of the icosahedron (Ih). Energy values are presented in $\mathrm{eV}$.

\begin{tabular}{cccc}
\hline \# of Cu atoms & Ih & Dh & Coh \\
\hline 13 & 0.0 & 0.73 & 0.24 \\
\hline 55 & 0.0 & 2.68 & 3.62 \\
\hline 147 & 0.0 & 5.38 & 7.00 \\
\hline
\end{tabular}

